\title{
Performance and energy systems contributions during upper-body sprint interval exercise
}

\author{
Emerson Franchini ${ }^{1}$, Monica Yuri Takito, ${ }^{2 *}$, Maria Augusta Peduti Dal'Molin Kiss ${ }^{3}$ \\ ${ }^{1}$ Martial Arts and Combat Sports Research Group, School of Physical Education and Sport, University of Sao Paulo, Sao Paulo, Brazil \\ ${ }^{2}$ Human Movement Pedagogy Department, School of Physical Education and Sport, University of Sao Paulo, Sao Paulo, Brazil \\ ${ }^{3}$ School of Physical Education and Sport, University of Sao Paulo, Sao Paulo, Brazil
}

The main purpose of this study was to investigate the performance and energy systems contribution during four upper-body Wingate tests interspersed by 3-min intervals. Fourteen well-trained male adult Judo athletes voluntarily took part in the present study. These athletes were from state to national level, were in their competitive period, but not engaged in any weight loss procedure. Energy systems contributions were estimated using oxygen uptake and blood lactate measurements. The main results indicated that there was higher glycolytic contribution compared to oxidative $(P<0.001)$ during bout 1 , but lower glycolytic contribution was observed compared to the phosphagen system (adenosine triphosphate-creatine phosphate, ATP-PCr) contribution during bout $3(P<0.001)$, lower glycolytic contribution compared to oxidative and ATP-PCr ( $P<0.001$ for both comparisons) contributions during bout 4 and lower oxidative compared to ATP-PCr during bout $4(P=0.040)$. For the energy system contribution across Wingate bouts, the ATP-PCr contribution during bout 1 was lower than that observed during bout 4 $(P=0.005)$, and the glycolytic system presented higher percentage contribution in the first bout compared to the third and fourth bouts $(P<0.001$ for both comparisons), and higher percentage participation in the second compared to the fourth bout $(P<0.001)$. These results suggest that absolute oxidative and ATP-PCr participations were kept constant across Wingate tests, but there was an increase in relative participation of ATP-PCr in bout 4 compared to bout 1, probably due to the partial phosphocreatine resynthesis during intervals and to the decreased glycolytic activity.

Keywords: Wingate tests, Glycolysis, Energy expenditure

\section{INTRODUCTION}

High-intensity interval training (HIIT) has gained great attention of the scientific literature in the last decades, mainly because of its excellent cost-benefit ratio concerning physiological adaptation and health-related physical fitness improvement in short period of time, and a variety of protocols have been used (Buchheit and Laursen, 2013a, 2013b). Among the different training protocols the sprint interval training, with intensities above $160 \%$ of the intensity associated to maximum oxygen uptake $\left(\mathrm{VO}_{2 \max }\right)$ up to the maximum sprint intensity, is considered the upper limit of HIIT (Buchheit and Laursen, 2013a). The use of this kind of protocol was found to improve both aerobic (maximal aerobic power) and anaerobic performance (peak and mean power in the second to fourth Wingate tests, with 4-min intervals), and to result in increased enzymatic activation for hexokinase, phosphofructokinase (PFK), malate dehydrogenase, succinate dehydrogenase and citrate synthase, after 7 weeks of 4 to $10 \times 30$-sec cycle ergometer sprints (Wingate tests) conducted 3 times per week (MacDougall et al., 1998).

Although there are references concerning the elevated glycolytic nature of the Wingate test (Granier et al., 1995; Serresse et al., 1988; Smith and Hill, 1991), few of these studies reported the energy system contributions to the upper-body version of this kind of exercise (Harvey et al., 2015; Lovell et al., 2013; Price et al., 2014), The energy systems contribution to the single lower-body Wingate test has been reported to be around $19 \%$ to $30 \%$ for the oxidative system, $23 \%$ to $31 \%$ for the phosphagen system (adenosine tri-
${ }^{*}$ Corresponding author: Monica Yuri Takito (iD http://orcid.org/0000-0003-0257-8765 Human Movement Pedagogy Department, School of Physical Education and Sport, University of São Paulo, Av Prof Melo Moraes, 65 São Paulo, SP 05508-900, Brazil Tel: +55-11-30912306, Fax: +55-11-30913135, E-mail: mytakito@gmail.com Received: September 13, 2016 / Accepted: December 10, 2016
This is an Open Access article distributed under the terms of the Creative Commons Attribution Non-Commercial License (http://creativecommons.org/licenses/by-nc/4.0/) which permits unrestricted non-commercial use, distribution, and reproduction in any medium, provided the original work is properly cited. 
phosphate-creatine phosphate, ATP-PCr) and $49 \%$ to $56 \%$ for the glycolytic system (Beneke et al., 2002; Granier et al., 1995; Serresse et al., 1988; Smith and Hill, 1991), while for upper-body Wingate test the values observed were: $11.4 \% \pm 1.4 \%, 60.3 \% \pm 5.6 \%$, and $28.3 \% \pm 4.9 \%$ for the oxidative, glycolytic, and ATP-PCr systems, respectively (Lovell et al., 2013). However, only two studies (Harvey et al., 2015; Price et al., 2014) compared the energy systems contributions in lower- and upper-body Wingate tests in the same participants. Price et al. (2014) observed a higher oxidative contribution to the upper-body version $(43.5 \% \pm 29.3 \%)$ compared to the lower-body one $(29.4 \% \pm 15.8 \%)$, while Harvey et al. (2015) reported that the oxidative system contribution was higher for the lower-body $(16.8 \% \pm 2.5 \%)$ compared to the upper body $(11.4 \% \pm 1.4 \%)$, the glycolytic contribution was higher during the upper-body $(60.3 \% \pm 5.6 \%)$ compared to the lower body $(46.9 \% \pm 6.9 \%)$, and the ATP-PCr contribution was higher during the lower body $(36.5 \% \pm 6.3 \%)$ compared to the upper-body $(28.3 \% \pm 4.9 \%)$ with Wingate test. Thus, the understanding of the energy systems contributions to the upper-body Wingate test is only beginning and to the best of our knowledge none investigate the energy systems responses to this sprint interval exercise using the upper-body. It is important to consider that basketball Paralympic athletes and athletes competing in Judo and Wrestling, for example, normally execute repeated high-intensity actions during their competitive disputes. The understanding of the energy system dynamics during high-intensity interval exercise is important to improve the comprehension of the stimulus generated and, consequently, of the probable long-term adaptation to such effort (Lira et al., 2015). Thus, the main objective of this study was to investigate the performance and energy systems contribution during four upper-body Wingate tests interspersed by 3-min intervals. The main hypothesis of this study was that the glycolytic pathway would decrease its contribution during the Wingate repetitions and that the oxidative system would partially compensate for this decrease.

\section{MATERIALS AND METHODS}

\section{Study design}

This is a descriptive and correlational study investigating the performance, physiological and energy systems estimated contribution to four bouts of upper-body Wingate tests with 3-min intervals in-between. Athletes were informed of all procedures and signed an informed consent form before participation. All procedures were approved by the local research ethic committee of
School of Physical Education and Sports (CAAE: 0060.0.342.00012). After anthropometric measurements athletes were submitted to the sprint interval exercise protocol, where oxygen uptake and heart rate measurements were conducted continuously during the exercise and interval periods up to $3 \mathrm{~min}$ after the last bout, while blood lactate measurements were conducted before and after each exercise bout.

\section{Sample}

Fourteen well-trained male adult Judo athletes voluntarily took part in the present study. These athletes were from state to national level, were in their competitive period, but not engaged in any weight loss procedure. They were instructed to maintain their habitual diet and not perform any vigorous exercise in the $24 \mathrm{hr}$ before the testing protocol. The main characteristics of this group are as follows (mean and standard deviation): age, $21 \pm 3$ years; height, $175.8 \pm 10.2 \mathrm{~cm}$; body mass, $75.4 \pm 15.5 \mathrm{~kg}$; body fat percentage (Drinkwater and Ross, 1980), 13.5 \pm 3.2 ; Judo training experience, $11 \pm 4$ years.

\section{Wingate tests}

Four Wingate test bouts were performed in a Monark (Vansbro, Sweden) cycle ergometer adapted to the upper-body, using 0.05 $\mathrm{kg} / \mathrm{kg}$ of athletes' body mass, as this load was found to be optimal to result in higher mean power during this protocol in Judo athletes. During the 3-min intervals between tests athletes remained seated in the cycle ergometer. Power output was registered using the Wingate test software (Cefise, Nova Odessa, Brazil), which allowed the power measurement for each second of the tests. Based on these data, relative peak (highest power generated during the test; $\mathrm{W} / \mathrm{kg}$ ) and mean power (average power during each 30-sec period; $\mathrm{W} / \mathrm{kg}$ ) were determined. These procedures were similar to that used in previous studies (Gaiga and Docherty, 1995; MacDougall et al., 1998).

\section{Physiological measurements}

Heart rate (Polar Electro Oy, Vantaa, Finland) and oxygen uptake (Cosmed K4b2, Rome, Italy) were measured continuously throughout the Wingate tests and during the intervals, including 3 min post the fourth bout, while blood lactate was measured before the first test and $1 \mathrm{~min}$ after each Wingate test using a Yellow Springs 1500 Sport (YSI Incorporated, Yellow Springs, OH, USA). The highest 5-sec heart rate and oxygen uptake values during each test were considered as heart rate peak and peak of oxygen uptake. Additionally, total oxygen uptake during each 
Wingate bout was determined. Before each test the gas analyzer was calibrated in accordance with the manufacturer's recommendations. Delta of blood lactate was calculated subtracting post minus pre Wingate test blood lactate concentration.

\section{Energy systems contributions}

Estimates of oxidative, glycolytic and ATP-PCr systems contributions were carried out through the measurement of oxygen uptake, blood lactate concentration and the fast phase of excess oxygen consumption after exercise, respectively. Briefly, oxidative energy was estimated by subtracting oxygen uptake at rest from that during each Wingate test by the trapezoidal method (i.e., a method to calculate the area under the curve). Rest oxygen uptake was determined in the seated position with the last $30 \mathrm{sec}$ of a 5-min period used as reference. The glycolytic pathway contribution was calculated assuming that the accumulation of $1 \mathrm{mmol} / \mathrm{L}$ of blood lactate is equivalent to $3 \mathrm{~mL}$ of oxygen $/ \mathrm{kg}$ of body mass (Di Prampero and Ferretti, 1999). The ATP-PCr contribution was estimated considering the fast component of the excess of oxygen consumption after each Wingate bout, which was fitted using the bi-exponential function as described by Beneke et al. (2002), via the use of Origin 6.0 software (Microcal Software, Northampton, MA, USA). The caloric quotient of $20.92 \mathrm{~kJ}$ (Gastin, 2001) was used in all three different energy systems and the total metabolic work was calculated as the sum of the three energy systems. Furthermore, the contribution of the three energy systems was also expressed in percentage of total energy expenditure.

\section{Statistics}

The data were analyzed using Statistica for Windows 12.0 software (StatSoft, Tulsa, Oklahoma, USA). The distribution of the data was analyzed using the Shapiro-Wilk test, which showed a normal Gaussian distribution. The descriptive analysis involved the calculation of the mean and standard deviation. The compound symmetry was verified and confirmed through the Mauch- ly test. The performance, total energy expenditure and physiological variables were compared using an one-way analysis of variance (ANOVA) with repeated measurements, while a two-way (system and Wingate test) ANOVA was used to compare the energy systems contributions throughout the tests. When a significant effect was found in the ANOVA, the Bonferroni multiple comparison test was used in order to identify possible differences between Wingate tests and energy systems. For the two-way ANOVA only interaction effects were presented and for post hoc comparisons only the differences between the systems in the same Wingate bout and for a given system in different Wingate bouts were reported. The eta squared $\left(\mathrm{n}^{2}\right)$ was used to quantify the effect size and classified using the following scale for interpretation (Hopkins, 2006): $<0.1$ (trivial), 0.1 to $<0.3$ (small), 0.3 to $<0.5$ (moderate), 0.5 to $<0.7$ (large) and 0.9 to 1.0 (perfect). Relationship between mean power, peak power and energy systems contributions was assessed via Pearson product correlation, considering each bout individually and all bouts grouped. The values of $\mathrm{r}$ were classified as suggested by Hopkins (2006): 0.0 to 0.1 (trivial), 0.1 to 0.3 (small), 0.3 to 0.5 (moderate), 0.5 to 0.7 (large), 0.7 to 0.9 (very large) and 0.9 to 1.0 (nearly perfect), and only correlations above large were reported. The statistical significance was set at $5 \%$.

\section{RESULTS}

An effect of Wingate bout was found for mean $\left(F_{3}, 39=62.02\right.$; $\left.P<0.001 ; \eta^{2}=0.827\right)$ and peak power $\left(F_{3}, 39=20.10 ; P<0.001\right.$; $\left.\eta^{2}=0.607\right)$. Athletes achieved higher mean power during bout 1 compared to all other bouts, and higher values during bout 2 compared to bouts 3 and 4 ( $P<0.001$ for all comparisons). For peak power, higher values were achieved during bout 1 compared to bouts 2 ( $P=0.048), 3$ and $4(P<0.001$ for both comparisons) and higher values on bout 2 compared to bout $4(P<0.001)$ (Fig. 1).

Table 1 presents the main physiological responses to the upper-body sprint exercise. Blood lactate increased $\left(F_{4}, 52=267.66\right.$;

Table 1. Physiological responses to four bouts of upper-body Wingate tests $(n=14)$

\begin{tabular}{|c|c|c|c|c|c|}
\hline Variable & Before & Wingate 1 & Wingate 2 & Wingate 3 & Wingate 4 \\
\hline $\mathrm{VO}_{2 \text { peak }}(\mathrm{mL} / \mathrm{kg} / \mathrm{min})$ & - & $48.79 \pm 10.04$ & $48.69 \pm 6.95$ & $47.49 \pm 8.81$ & $49.83 \pm 9.22$ \\
\hline $\mathrm{VO}_{2}(\mathrm{~mL})$ & - & $858 \pm 323^{b, c, d)}$ & $1,091 \pm 257$ & $1,079 \pm 225$ & $1,056 \pm 269$ \\
\hline Heart rate peak (bpm) & - & $172 \pm 13$ & $174 \pm 10$ & $172 \pm 10$ & $173 \pm 12$ \\
\hline Lactate (mmol/L) & $1.32 \pm 0.60^{a, b, c, d)}$ & $6.66 \pm 1.45^{b, c, d)}$ & $10.55 \pm 1.57^{\mathrm{c}, \mathrm{d})}$ & $12.37 \pm 1.63$ & $13.47 \pm 1.93$ \\
\hline Delta lactate (mmol/L) & - & $5.34 \pm 1.82^{b, c, d l}$ & $3.88 \pm 1.00 c^{d l}$ & $1.83 \pm 0.93$ & $0.88 \pm 0.74$ \\
\hline Energy expenditure (kJ) & - & $55.76 \pm 15.50^{c, d)}$ & $52.67 \pm 10.13^{c, d)}$ & $40.19 \pm 11.46$ & $34.98 \pm 13.45$ \\
\hline
\end{tabular}

Values are presented as mean \pm standard deviation.

a,b,c,d) Different from bouts $1,2,3$, and 4 , respectively $(P<0.05)$. 


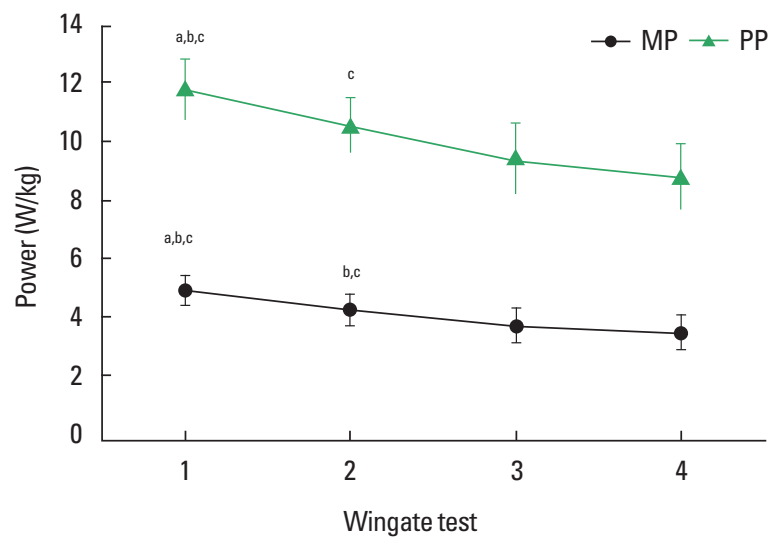

Fig. 1. Mean (MP) and peak power (PP) during 4 upper-body Wingate bouts $(n=14)$. Values are mean and standard deviation. ${ }^{a, b, c i}$ Different from bouts 2,3 , and 4 , respectively $(P<0.05)$.

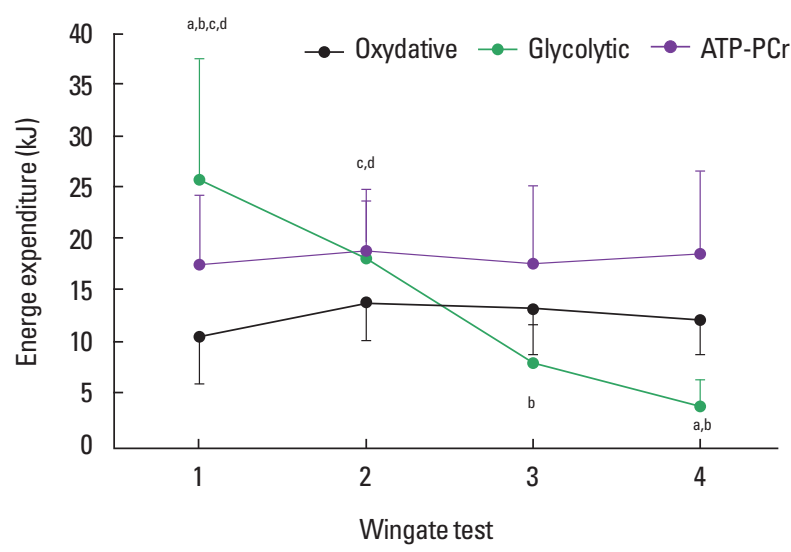

Fig. 2. Absolute (kJ) energy systems contributions during four bouts of upper-body Wingate tests $(n=14)$. Values are mean and standard deviation. ${ }^{a} D$ ifferent from oxidative contribution in the same bout $(P<0.05)$. ${ }^{\text {b Different from }}$ adenosine triphosphate -creatine phosphate (ATP-PCr) contribution in the same bout $(P<0.05)$. ${ }^{c}$ Different from bout three for the same system $(P<0.05)$. ${ }^{\text {dI }} D$ ifferent from bout four for the same system $(P<0.05)$.

$\left.P<0.001 ; \eta^{2}=0.954\right)$ from rest to post each Wingate bout, from bout 1 to all other bouts and from bout 2 to bouts 3 and 4 ( $P<0.001$ for all comparisons). An opposite change $\left(F_{3}, 39=34.76\right.$; $P<0.001 ; \eta^{2}=0.728$ ) was observed for delta of blood lactate, i.e., a higher delta was found for bout 1 compared to bouts $2(P=0.026)$, 3 and 4 ( $P<0.001$ for both comparisons), and higher values for bout 2 compared to bouts 3 and $4(P<0.001$ for both comparisons). Conversely, $\mathrm{VO}_{2 \text { peak }}\left(F_{3}, 39=0.32 ; P=0.811 ; \eta^{2}=0.024\right)$ and heart rate peak $\left(F_{3}, 39=0.69 ; P=0.565 ; \eta^{2}=0.059\right)$ did not change across Wingate bouts. However, total oxygen uptake was affected by Wingate bouts $\left(F_{3}, 39=6.83 ; P<0.001 ; \eta^{2}=0.345\right)$, with lower values in the first bout compared to bouts $2(P=0.002), 3$ $(P=0.004)$, and $4(P=0.011)$. There was also an effect of Wingate

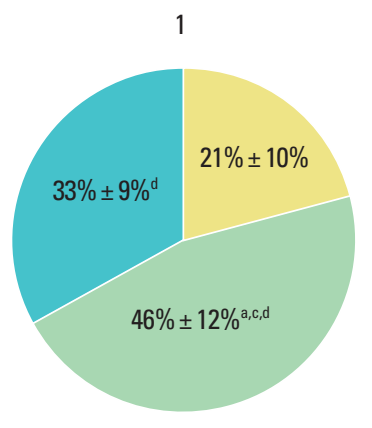

3

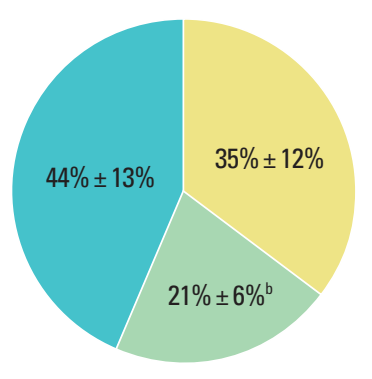

Oxidative

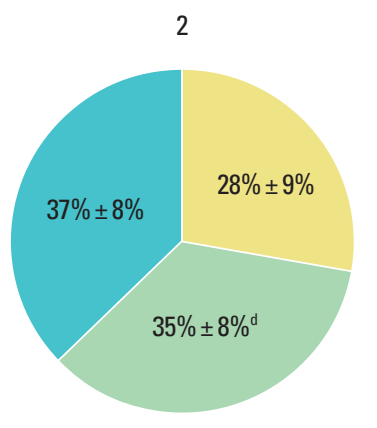

4

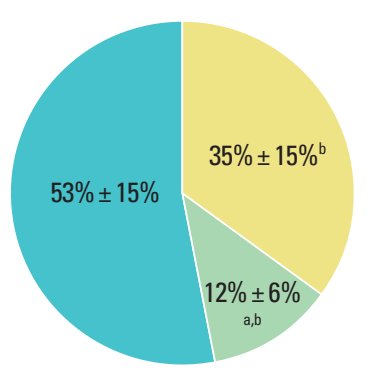

Glycolytic ATP-PCr

Fig. 3. Relative (\%) energy systems contributions during four bouts of upper-body Wingate tests $(n=14)$. Values are mean and standard deviation. ${ }^{\text {al } D i f-~}$ ferent from oxidative contribution in the same Wingate test $(P<0.05)$. ${ }^{\text {b }}$ Different from ATP-PCr contribution in the same bout $(P<0.05)$. ${ }^{\mathrm{c}}$ Different from bout three for the same system $(P<0.05)$. ${ }^{\text {dif } D i f f e r e n t ~ f r o m ~ b o u t ~ f o u r ~ f o r ~ t h e ~ s a m e ~}$ system $(P<0.05)$.

bouts on energy expenditure $\left(F_{3},{ }_{39}=12.41 ; P<0.001 ; \eta^{2}=0.488\right)$, with higher values during bouts 1 and 2 compared to bouts 3 $(P=0.001$ and $P=0.015$, respectively) and $4(P<0.001$ for both comparisons).

For the absolute energy expenditure (Fig. 2) there was an interaction effect $\left(F_{6}, 78=15.92 ; P<0.001 ; \eta^{2}=0.551\right)$. Concerning the comparison between energy systems in the same Wingate bout, there was higher glycolytic contributions compared to oxidative and ATP-PCr $(P<0.001$ and $P=0.045$, respectively) during bout 1 , but a lower glycolytic contribution was observed compared to the ATP-PCr contribution during bout $3(P=0.006)$, and lower glycolytic contribution compared to oxidative $(P=0.033)$ and ATP-PCr $(P<0.001)$ contributions during bout 4 . For the energy system contribution across Wingate bouts, only the glycolytic system had its participation decreased along the tests, with higher values in the first and second bouts compared to the third ( $P<0.001$ and $P=0.002$, respectively) and fourth bouts $(P<0.001$ for both comparisons).

For the relative energy expenditure (Fig. 3) there was an interaction effect $\left(F_{6}, 78=16.34 ; P<0.001 ; \eta^{2}=0.557\right)$. Concerning the 
comparison between energy systems in the same Wingate bout, there was higher glycolytic contribution compared to oxidative $(P<0.001)$ during bout 1 , but lower glycolytic contribution was observed compared to the ATP-PCr contribution during bout 3 $(P<0.001)$, lower glycolytic contribution compared to oxidative and ATP-PCr $(P<0.001$ for both comparisons) contributions during bout 4 and lower oxidative compared to ATP-PCr during bout $4(P=0.040)$. For the energy system contribution across Wingate bouts, the ATP-PCr contribution during bout 1 was lower than that observed during bout $4(P=0.005)$, and the glycolytic system had its participation decreased along the tests, with higher percentage contribution in the first bout compared to the third and fourth bouts $(P<0.001$ for both comparisons), and higher percentage participation in the second compared to the fourth bout $(P<0.001)$.

Significant correlations $(P<0.05)$ were found between: mean power and absolute $(r=0.80)$ and relative $(r=0.54)$ glycolytic contribution during bout 1 ; mean power and absolute ATP-PCr contribution during bouts $1(r=0.59), 2(r=0.55)$, and $3(r=0.66)$; peak power and absolute ATP-PCr contribution during bouts 1 $(r=0.61), 2(r=0.55)$, and $3(r=0.59)$; peak power and absolute $(r=0.77)$ and relative $(r=0.55)$ glycolytic contribution during bout 1; percentage of mean power decline and percentage of glycolytic decline along the four bouts $(r=0.53)$.

When all data was grouped, mean power was significantly correlated $(P<0.001)$ to absolute glycolytic contribution $(r=0.64)$ and total energy expenditure $(r=0.71)$, while peak power was significantly correlated $(P<0.001)$ to absolute glycolytic contribution $(r=0.51)$ and total energy expenditure $(r=0.62)$.

\section{DISCUSSION}

The main findings of this study was that there was a mean and peak power decrease along Wingate tests repetitions, which was accompanied by a decrease in absolute and relative glycolytic contribution. Absolute oxidative and ATP-PCr participations were kept constant across Wingate tests, but there was an increase in relative participation of ATP-PCr in bout 4 compared to bout 1 .

The decrease in mean and peak observed in the present study is similar to other studies that investigating similar protocols using lower-body Wingate tests (McCartney et al., 1986; Trump et al., 1996), including those with Judo athletes (Kim et al., 2011) and wrestlers (Farzad et al., 2011). This decline in performance is normally described as consequence of the decreased glycolytic activation across repeated sprint bouts (McCartney et al., 1986; Trump et al., 1996), which was confirmed by the correlation observed in our study concerning the percentage decrement in mean power and glycolytic contribution. Although the phosphocreatine resynthesis during the recovery period is important to avoid further performance impairment (Bogdanis et al., 1996), and an increased oxygen uptake was observed in the last bout compared to the first one, these metabolic pathways were not able to keep exercise intensity during this exercise, as a significant decrease in total energy expenditure was observed in the last two compared to the first two bouts. Traditionally, the decrease in the glycolytic activation has been considered to be due to the PFK inhibition caused increased $\mathrm{H}^{+}$, which would affect the glycolytic flux and result in lower adenosine triphosphate (ATP) resynthesis (Cairns, 2006).

As the absolute contribution of the oxidative and ATP-PCr pathways did not change across the sprints and the glycolytic system decreased its participation, the relative energy system contribution changed across the bouts. In the first bout it was observed a predominance of the glycolytic system in relation to the other two pathways, which is similar to findings reported in studies investigating lower-body Wingate test (Beneke et al., 2002; Granier et al., 1995; Serresse et al., 1988; Smith and Hill, 1991), but differed from the studies that used the upper-body protocol (Harvey et al., 2015; Lovell et al., 2013; Price et al., 2014). Basically, our athletes presented higher oxidative contribution compared to those reported by Lovell et al. (2013) and Harvey et al. (2015), but lower than those observed by Price et al. (2014), lower glycolytc activation compared to those estimated by Lovell et al. (2013) and Harvey et al. (2015) and similar ATP-PCr contribution as in other studies (Harvey et al., 2015; Lovell et al., 2013). The fact that our sample was composed by highly-trained Judo athletes can partially explain these differences. As Judo involves many upper-body actions (Franchini et al., 2013), probably our group had well-developed aerobic fitness in this region, which resulted in higher oxidative contribution during the first bout when compared to other groups previously studied. The decrease in the glycolytic contribution along the Wingate tests, the partial resynthesis of phosphocreatine in the intervals (Bogdanis et al., 1996) and the increased oxygen uptake in the last bout, explain why the glycolytic pathway presented a lower percentage participation compared to the ATP-PCr system in the last two bouts and lower than the oxidative system in the last bout. The increased ATP-PCr relative contribution in the fourth sprint compared to the first is similar to the response observed by Gaitanos et al. (1993) using ten 6-sec sprints interspersed by $30 \mathrm{sec}$ of passive recovery, which indicated that $49.6 \%$ participation of phosphocreatine in the first 
bout and $80.1 \%$ contribution in the tenth bout. Basically, the phosphocreatine resynthesis during the intervals (around 75\% considering the data from Bogdanis et al., 1996) was able to compensate for the depletion during the previous bout and to provide enough energy to keep its absolute participation constant in the four bouts; as the glycolytic system decreased its participation, the relative ATP-PCr participation increased.

In summary, our findings indicated decreased mean and peak power throughout the four upper-body Wingate tests, which was related by decreased absolute and relative glycolytic contribution. Absolute oxidative and ATP-PCr participations were kept constant across Wingate tests, but there was an increase in relative participation of ATP-PCr in bout 4 compared to bout 1, probably due to the partial phosphocreatine resynthesis during intervals and to the decreased glycolytic activity.

\section{CONFLICT OF INTEREST}

No potential conflict of interest relevant to this article was reported.

\section{ACKNOWLEDGMENTS}

This work was supported by the FAPESP (Fundação de Amparo à Pesquisa do Estado de São Paulo) (2012/00220-8). The first author is supported by a CNPq grant (302242/2014-7).

\section{REFERENCES}

Beneke R, Pollmann C, Bleif I, Leithäuser RM, Hütler M. How anaerobic is the Wingate Anaerobic Test for humans? Eur J Appl Physiol 2002;87: 388-392.

Bogdanis GC, Nevill ME, Boobis LH, Lakomy HK. Contribution of phosphocreatine and aerobic metabolism to energy supply during repeated sprint exercise. J Appl Physiol (1985) 1996;80:876-884.

Buchheit M, Laursen PB. High-intensity interval training, solutions to the programming puzzle: Part I: cardiopulmonary emphasis. Sports Med 2013a;43:313-338.

Buchheit M, Laursen PB. High-intensity interval training, solutions to the programming puzzle. Part II: anaerobic energy, neuromuscular load and practical applications. Sports Med 2013b;43:927-954.

Cairns SP. Lactic acid and exercise performance: culprit or friend? Sports Med 2006;36:279-291.

di Prampero PE, Ferretti G. The energetics of anaerobic muscle metabolism: a reappraisal of older and recent concepts. Respir Physiol 1999;
118:103-115.

Drinkwater DT, Ross WD. Anthropometric fractionation of body mass. In: Ostyn M, Beunen G, Simons J, editors. Kinanthropometry II. Baltimore (MD): University Park Press; 1980. p.177-189.

Farzad B, Gharakhanlou R, Agha-Alinejad H, Curby DG, Bayati M, Bahraminejad M, Mäestu J. Physiological and performance changes from the addition of a sprint interval program to wrestling training. J Strength Cond Res 2011;25:2392-2399.

Franchini E, Artioli GG, Brito CJ. Judo combat: time-motion analysis and physiology. Int J Perform Anal Sport 2013;13:624-641.

Gaiga MC, Docherty D. The effect of an aerobic interval training program on intermittent anaerobic performance. Can J Appl Physiol 1995;20: 452-464.

Gaitanos GC, Williams C, Boobis LH, Brooks S. Human muscle metabolism during intermittent maximal exercise. J Appl Physiol (1985) 1993; 75:712-719.

Gastin PB. Energy system interaction and relative contribution during maximal exercise. Sports Med 2001;31:725-741.

Granier P, Mercier B, Mercier J, Anselme F, Préfaut C. Aerobic and anaerobic contribution to Wingate test performance in sprint and middle-distance runners. Eur J Appl Physiol Occup Physiol 1995;70:58-65.

Harvey L, Wiegand A, Solomon C, Mclellan C, Lovell D. A comparison of upper and lower body energetics during high-intensity exercise. J Sports Med Phys Fitness 2015;55:708-713.

Hopkins WG. A scale of magnitudes for effect statistics: a new view of statistics [internet]. Internet Society of Sports Science; 2006 [updated 2006 Aug 7; cited 2013 Jun 28]. Available from: http://www.sportsci. org/resource/stats/effectmag.html

Kim J, Cho HC, Jung HS, Yoon JD. Influence of performance level on anaerobic power and body composition in elite male judoists. J Strength Cond Res 2011;25:1346-1354.

Lira FS, Panissa VL, Julio UF, Franchini E. Differences in metabolic and inflammatory responses in lower and upper body high-intensity intermittent exercise. Eur J Appl Physiol 2015;115:1467-1474.

Lovell D, Kerr A, Wiegand A, Solomon C, Harvey L, McLellan C. The contribution of energy systems during the upper body Wingate anaerobic test. Appl Physiol Nutr Metab 2013;38:216-219.

MacDougall JD, Hicks AL, MacDonald JR, McKelvie RS, Green HJ, Smith $\mathrm{KM}$. Muscle performance and enzymatic adaptations to sprint interval training. J Appl Physiol (1985) 1998;84:2138-2142.

McCartney N, Spriet LL, Heigenhauser GJ, Kowalchuk JM, Sutton JR, Jones NL. Muscle power and metabolism in maximal intermittent exercise. J Appl Physiol (1985) 1986;60:1164-1169.

Price M, Beckford C, Dorricott A, Hill C, Kershaw M, Singh M, Thornton I. Oxygen uptake during upper body and lower body Wingate anaer- 
obic tests. Appl Physiol Nutr Metab 2014;39:1345-1351.

Serresse O, Lortie G, Bouchard C, Boulay MR. Estimation of the contribution of the various energy systems during maximal work of short duration. Int J Sports Med 1988;9:456-460.

Smith JC, Hill DW. Contribution of energy systems during a Wingate power test. Br J Sports Med 1991;25:196-199.

Trump ME, Heigenhauser GJ, Putman CT, Spriet LL. Importance of muscle phosphocreatine during intermittent maximal cycling. J Appl Physiol (1985) 1996;80:1574-1580. 